\title{
Xerography in Card Reproduction
}

\author{
Mr. Dawson is assistant director, Univer- \\ sity of Chicago Library.
}

$\mathrm{T}$ HE REPRODUCTION of catalog cards has been a persistently annoying problem since libraries began using card catalogs. At first, cards were laboriously written in library hand; then, with the introduction of the typewriter, as many cards as were required were typed individually, a practice still employed by some libraries. Cards have been printed by libraries, usually by the use of the Multigraph, though sometimes on a job press. The Library of Congress, of course, continues to print its cards for its own catalogs and for distribution to other libraries; but most libraries which printed their cards found the cost of setting type and distributing it too expensive. Even when the type was not distributed after printing but was sold as metal, still the cost of typesetting and of buying new type was exorbitant for the limited number of copies run off.

The next step after the introduction of the typewriter was the adaptation of various duplicating processes to the reproduction of catalog cards. Experiments with gelatin reproduction processes were generally unsuccessful; the analine dyes used in the ink were of an unpleasant color, and, more serious, they soon faded; the images were fuzzy, and results were uneven and uncertain.

The use of stencils proved much more successful, and many libraries now rely on this process with improved stencils, inks, and duplicating machines. A variation of the stencil method is that employed in some public libraries where stencils normally used for addressing envelopes are used. ${ }^{1}$ However, the limited space available for text on these stencils restricts the use of this method largely to fiction, or to other materials requiring only brief cataloging, so that it is not feasible for research libraries.

Many libraries have, in recent years, turned to the special offset presses which were designed mainly for office use and which are simple enough to be operatedafter a brief training period-by unskilled personnel. These office offset presses use paper mats on which copy is typed or printed, or photographed (on photosensitive mats), and employ a permanent ink, similar to the ink used in regular printing, which produces clear images. One difficulty encountered in using this method is that the ink, having an oil base, is not readily absorbed by the smooth surface of the 100 per cent rag card stock, which sometimes causes smearing or the offsetting of the image from one card onto the back of another card during the long period before the ink has completely dried. However, using a quick drying ink or a combination of ink and a drying compound with the ink appears to eliminate this difficulty. The Library of Congress prints offset mats when printing its cards so that when the original stock is exhausted additional copies may be inexpensively reproduced by offset printing.

One of the most promising means for card reproduction which has recently been developed is the combination of xerography ${ }^{2}$

\footnotetext{
1 Bowers, M. E: "Cataloging with Stencils," Library Journal, 65:462-63, June I, I940.

2 Xerography, or to use the trade name, XeroX, was 2 Xerography, or, to use the trade name, XeroX, was
developed by the Battelle Memorial Institute and the Haloid Corporation. A discussion of xerography in libraries and a list of articles on it may be found in a
} 
and offset printing. Xerography is an electrical and mechanical rather than a chemical method of reproduction, as the various photographic methods are. Instead of a photographic negative, xerography employs an aluminum plate covered with an extremely thin coating of selenium, a "photoconductive" material. The peculiar property of selenium, which is also the one that makes xerography possible, is that it conducts electricity only when exposed to light; in the dark, it is a nonconductor.

In xerography, the selenium covered aluminum plate is sprayed with an electrostatic charge. In a camera unit, the plate is exposed to the material to be copied. As light is reflected from the white surfaces of the copy onto the charged selenium, the selenium becomes a conductive material and discharges the electrostatic charge into the aluminum part of the plate; but the dark surfaces, i.e., the writing, drawing, or typing, do not reflect the light, so that there is a pattern of dark on the selenium plate, and the selenium remains a nonconductor and retains its electrostatic charge. There is, then, in effect, an invisible pattern of electrostatically charged selenium which is a mirror image of the material to be copied. The plate is kept from the light and a dry developing powder is cascaded over it. Particles of the powder are attracted to and held by the electrostatic charge. Now the plate may be exposed to light, and a mirror image of the original copy can be seen on the plate.

Next, a sheet of paper or a paper offset mat is placed on the plate, and is smoothed down so that the paper is in contact with the plate. The paper is then sprayed with an electrostatic charge which attracts the powder from the surface of the plate. When the paper is removed from the plate, a true

pamphlet edited by Hodgson, James G., The Use of Xerography in Libraries. Fort Collins, Colorado, Colorado A. \& M. College Library, I952. A new edition was issued in 1953 . image of the material copied can be seen. At this point, the powder is held to the paper only by the electrostatic charge, so that, if desired, unwanted parts of the copy can be wiped off. The paper is then inserted into an oven which fuses the powder onto the paper in a permanent reproduction of the original copy. A sheet of copy or a strip of cards can be reproduced in from three to four minutes.

If the powder has been transferred to a sheet of paper, only one copy will have been produced. If, however, the powder has been transferred to an offset mat, as many copies as are required may be obtained when the mat is run on the offset press. It is this combination of xerography and offset printing which is of interest in card reproduction.

\section{USE AT CHICAGO}

For many years, the catalogers at the University of Chicago Library have typed their copy as they catalog material, thus providing the typists with clear and legible copy from which to type stencils or offset mats. A study of the copy slips (or cards) showed that approximately 95 per cent of these cards were free from typographic errors and could be reproduced without alteration or correction. ${ }^{3}$ That meant that 95 per cent of the copy produced by the catalogers could be reproduced by the xerography-offset process. Since typists were difficult to obtain, expensive to train, and had a high rate of turnover, this method seemed to hold promise for relieving an unfortunate situation. It seemed, too, that this might well be a faster and more economical method than having the copy typed on mats and printed by offset, since it eliminated the need for proofreading and correcting the typist's work.

We therefore began experimenting with

3 The catalogers are not considered to be typists and no effort is or wrll be made to urge them to produce perfect copy. Where copy is not suitable for reproduction, it is sent to a typist to copy on an off set master. 
xerography-offset for reproducing our cata$\log$ cards. In studying the production of cards by offset, we had concluded that reproducing one set of cards at a time was not efficient, and that, with the equipment we had, the most efficient way was to print four sets at a time. ${ }^{4}$ To do this, we had typed copy for four cards on half an offset mat cut vertically, and reproduced them'on strips of card stock cut according to our specifications, $30 \mathrm{~cm}$. by $12.5 \mathrm{~cm}$., with four holes drilled at the appropriate places. A relatively simple homemade jig on a guillotine paper knife made it possible to cut these strips accurately into four cards $7.5 \mathrm{~cm}$. by $12.5 \mathrm{~cm}$., the standard size of catalog cards. It was obvious that it would be equally advantageous to reproduce four sets of cards simultaneously by xerographyoffset.

The $\mathrm{XeroX}$ camera unit is equipped with somewhat primitive registration guides, totally inadequate for the precision required in copying catalog cards. A simple guide made of two pieces of plastic forming a right angle was devised and affixed to the camera unit. A piece of stiff binders board was cut with true corners to fit on the camera unit, and two strips of masking tape with adhesive on both sides were pressed onto this board, so placed that when cards are placed on these strips, accurate registration of the images on the xerographic plates is possible when the board is placed within the guides affixed to the camera.

This process of reproducing catalog cards requires, then, these steps:

I. Sorting the cards produced by the catalogers to remove those which require retyping, and, at the same time, sorting these cards by the number of copies required.

2. Placing the cards on the registration board and placing this board on the camera unit.

${ }^{4}$ We have a Multilith model 75 . Some libraries with a Multilith model I 250 or similar equipment reproduce eight sets of cards at a time.
3. Making an offset mat by xerography as described above. The lines between the cards and the holes in the cards cast shadows which would be reproduced if they were not removed. They can be removed at either of two different stages in the process: from the plates before the image is transferred to the mat, or from the mat before the image is fused. Cotton swabs have been found most satisfactory for this purpose.

4. Printing by offset the required number of strips of catalog cards.

5. Cutting the strips into four parts. (The strips for more than one set of cards can, of course, be cut at the same time.)

After determining that the xerographyoffset method of card reproduction was technically satisfactory, the questions which required answering were: How much does it cost? Do we save time? If so, how much? Records were kept of the time required for the operations involved in both processes over a period of time, and the cost of labor and materials divided by the number of units (the number of sets of cards) produced. In estimating the cost of rental of the XeroX equipment (it is available only on a rental basis), the monthly cost was divided by the average number of sets of cards reproduced per month.

We had already determined that the cost of producing ten copies of a card by typing and offset printing was 23.3 cents, and required about 7.8 minutes of labor, exclusive of sorting by number of copies required.

Our study of the cost of the xerographyoffset method indicated that the cost for ten copies of a single card was 20.8 cents, and that 2.7 minutes of labor (again excluding the sorting by number of copies required) were required. These costs were determined on the basis of our written cards only, and the cost differential was not great. The labor differential is substantial, and the relatively high cost of the xerography-offset process, in relation to the labor involved, is due to the fixed rental charge 
which must be apportioned to the number of sets reproduced. If we reproduce LC cards ${ }^{5}$ from our proofsheet file and our old depository catalog, the cost of the reproduction of our written cards decreases from 20.8 cents to approximately 16.8 cents for ten copies of a card.

The cost of reproducing LC cards is the same as the cost of reproducing our own written cards, or 16.8 cents per set of ten copies. This is considerably less than the cost of ordering a set of ten LC cards, which, if ordered by number, would cost 6 cents for the first card and 3.5 cents for each additional card, a total of 37.5 cents. This 37.5 cents cost does not, of course, include the cost of ordering the cards. Since the proofsheet or depository card must be found in either case (since we order by card number), this cost is the same whether we reproduce LC cards or order them. The actual cost of placing the order and receiving and sorting the cards when they are returned costs us approximately 1.2 cents per set of cards. The cost of ordering LC cards, then, excluding the determination of the card number, is 38.7 cents, in contrast to our cost of I6.8 cents (again excluding the locating of the proofsheet or depository card). There is another economy: when the LC proofsheet goes to the cataloger the call number is added and corrections made, so that, when the proofsheet is reproduced, all cards have call number and corrections on them; whereas the call number and the corrections must be added to each card when the set is obtained from the Library of Congress.

It must be noted that in the cost figures given above, not all factors are accounted

5 “. . . anyone is free to copy LC catalog cards, adapt them, use them, or even sell them. There is no copyright on them and LC retains no literary rights." LC Information Bulletin, V, I $2:$ No.22, p. I2, June I, I953. for, and these costs in no instance reflect the full cost of sets of cards. In no instance is overhead, either direct or indirect, included, nor is the depreciation of equipment included. These elements are common to each of the processes and procedures and have not been considered.

In determining the cost of the XeroX rental per set of cards, the monthly rental fee was divided by the normal number of sets of cards reproduced each month. Actually, the rental should be divided by the number of exposures made each month, whether these exposures are for card reproduction or for the reproduction of any other type of material. This, of course, will tend to reduce the cost of card reproduction by xerography-offset since the share of the rental allotted to card reproduction will be less.

This method of card reproduction is not recommended without reservation. In the University of Chicago Library, the fact that the catalogers type their copy cards is a factor in the success of our operation. If typewriters had to be acquired and catalogers trained to type their copy, it is doubtful that the method would be advantageous for some time. Secondly, the rental fee for $\mathrm{Xero} X$ equipment is substantial (our equipment rents for $\$ 60$ per month, but newer models and more flexible camera units have higher rental fees), and a large volume of work is required to reduce the unit cost to reasonable rates for libraries.

The xerography-offset method has been in regular use at the University of Chicago Library since October, I952. It is a normal part of our operations, and has enabled us to eliminate a backlog of cards awaiting reproduction and to speed up the reproduction of cards in spite of an acute shortage of typists. 